\title{
Method of projection dynamic thermal tomography (PDTT)
}

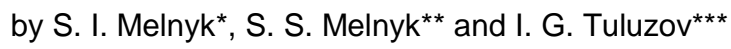

* Kharkiv National University of Radio Electronics, Lenin Ave.14, 61166, Kharkiv Ukraine smelnyk@yandex.ru

** A. Usikov Institute of Radio Physics and Electronics (NASU), Proskura str. 12, Kharkiv, 61085, Ukraine

*** Kharkiv Regional Centre for Investment, of.405, Tobolska str.12, Kharkiv, 61072, Ukraine

\begin{abstract}
The projection dynamic thermal tomography method is applicable for testing of objects with irregular internal structure. It allows reliably detecting the foreign inclusion-type defects and controlling with high accuracy the geometric parameters of the internal structure of objects. It is designed for using modern IR cameras with a standard set of parameters and does not require expensive additional equipment. Preliminary numerical analysis of capabilities of the proposed method for specific objects and the parameters of expected defects is possible, indicating the requirements to the parameters of IR camera and heating. This method is superior in terms of information value to alternative methods.
\end{abstract}

\section{Introduction}

The main problem of the existing methods of the dynamic thermal tomography (DTT) is the inhomogeneity of the emitting capability of the surface of the object of testing. For its solution in existing methods the phase shift $\varphi_{i k}$ between the harmonic thermal flow $q=q_{0} \sin w t$ and the surface temperature in each pixel of the thermal image $T(t)_{i k}=T_{0 i k} \cdot \sin \left(w t+\varphi_{i k}\right)$ is directly measured or calculated. Each value of $\varphi_{i k}$ is recalculated for the depth of defect location in the specific pixel of the thermogram using a simple analytical formula. According to the alternative method, the time characteristics of response for pulse action are measured. In all of the known examples [1-5] it is impossible to obtain a sufficiently high resolution for the deeply located detects. For detecting deeply located inhomogeneities the lowfrequency harmonic thermal action on the object's surface is required, which results in a significant (up to several minutes and hours) increase of the control time.

The results of a comparative analysis of the existing methods of DTT [4] show that;

1. They are mainly applicable to subsurface defects like cracks or delamination, and cannot ensure reconstruction of three-dimensional image of the internal structure of the tested object.

2. Deeply located objects cannot be detected using standard heating equipment.

3. Inaccuracy of detection of defect edges (or its size) is several times larger than the depth of its location.

4. The method of heating of objects requires special purpose equipment. Its parameters must be optimized with account of specific properties of the object of testing.

These drawbacks do not allow fully using the information about the object of testing obtained using the highly sensitive rapid-action thermal imaging devices. The reason of this effect is the rapid (diffusion) nature of the spreading of temperature perturbation caused by local inhomogeneities. At the same time, the characteristic spot size of the thermal anomaly on the surface is not less than the depth of occurrence of the defect. Therefore, the one-dimensional algorithms of reconstruction used in the known methods are efficient only at low (compared to the size of inhomogeneity) depths. In the method proposed by us, the following means are used for the reconstruction of the smoothed informative field:

1. We change the effective direction of the heat flow from perpendicular to the observed surface to parallel.

2. We use mathematical algorithms of two- and three-dimensional reconstruction, effectively taking account of the heat exchange between the areas of object corresponding to adjacent pixels of the thermal image.

3. We use the additive algorithms of information accumulation from several (minimum of two) consecutive measurements with various directions of effective heat flows.

\section{Structure of PDTT}

Unlike DTT, any method of object heating creating non-stationary thermal flows in various directions can be used. Thus, the dynamic thermal projections on the observed surface are formed. One of the possibilities of creating a thermal flow using contact method (heated support) in two orthogonally related directions is shown in the figure 1 . The highest information value is achieved in orthogonally related flows, similar to other methods of projective tomography. No special heating time mode is required, however the availability of complete information on the dynamics of thermal projections of the internal structure of the object on the observed surface is of principal importance. The minimum time required for PDTT is determined both by the parameters of the object of testing and the sensitivity and performance of the thermal imaging device. Complete cooling of the object is not required for repeated testing. The testing data are of additive nature (can complement the previously obtained data and improve the quality of reconstruction). In particular, 
upon completing the preliminary (coarse) detection of inhomogeneities in the object of testing, it is possible to create more intensive non-stationary thermal flows locally in the defect area, for instance, using directed laser emission.

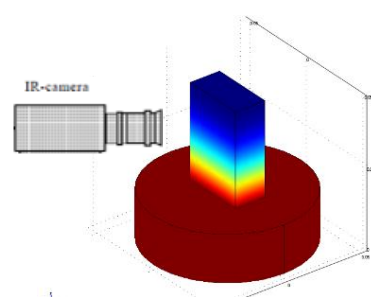

a

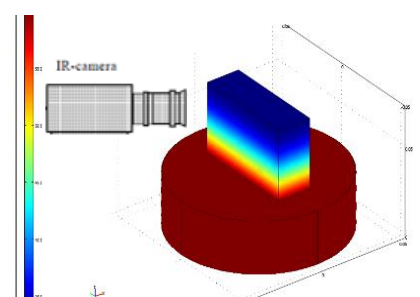

$b$

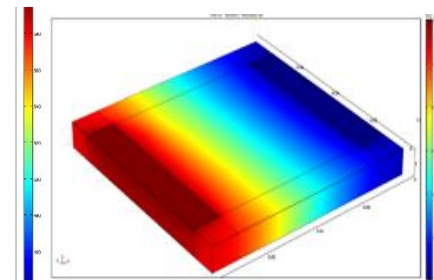

C

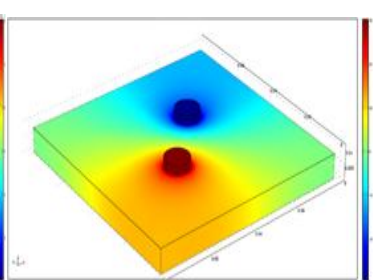

$d$

Fig. 1. (a, b) One of the possibilities of creating a thermal flow using contact method (heated support); (c, d) the method of creating a thermal flow using the one side heating (by the laser beam for example)

In case of random nature of heating the measured thermal emission flow depends not only on the real surface temperature, but also on its emissivity factor. Unlike the known DTT methods, the filtration of artifacts requires taking account of the dynamics of the whole process. Such processing is performed in each pixel of the thermal image and the dynamics of measurements in adjacent pixels is also taken into account. The accuracy of the filtration is determined both by the number of adjacent pixels taken into account and the completeness of the obtained measurement information. Fig. 2 shows the example of such reconstruction in case of film processing in pixel with correct (2a) and incorrect (2b) adjustment of the emissivity factor in the thermal imager parameters. The calculation was made for 400 frames of the control procedure modeled in the finite element model. It is clear from the figure that all points are located along the straight line, at the same time the line crosses the origin of coordinates only for correct values of the emissivity factor. Thus, the dynamical analysis of the thermal image allows determining both the distribution of the emissivity factor on the observed surface and the real value of its temperature with great accuracy.

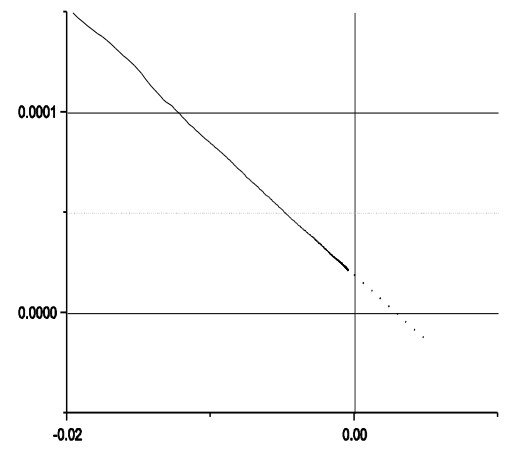

a

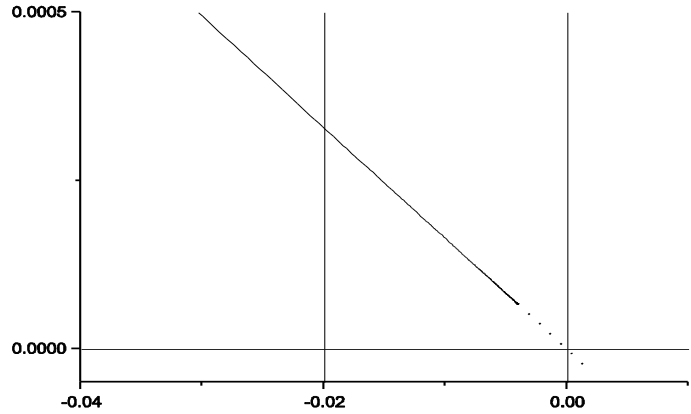

$b$

Fig. 2. Dependency of model parameters for 400 consecutive frames of thermofilm (a) - on the inhomogeneity of the coefficient of surface emission, (b) on the inhomogeneity of the internal profile.

The further reconstruction is performed for the values of surface temperature already corrected for the whole thermofilm. Forming the three dimensional image of the object's internal structure is achieved by using special algorithms of reconstruction based on the analysis of thermofilms. The reconstruction process includes three stages.

1. Construction of the approximate profile of the deeply-located inhomogeneity as a result of solution of the two-dimensional inverse problem of thermal conductivity.

2. Specification of the obtained profile using the algorithm of focusing. In this case, an analog of the "focusing screw" (similar to a real microscope) is present in the algorithm - a parameter providing the most accurate reconstruction of the profile at the set depth.

3. Algorithm of account of the a priori information on characteristic features of the required profile. Such features can be the parameters of its smoothness, amplitude, etc. Using a priori information on the parameters of the object's internal structure allows increasing its sensitivity in several times [6].

In the following chapter we will illustrate the results of reconstruction of inhomogeneities using only the first two algorithms. Using the third aalgorithm provides an even greater increase of the resolution of the method depending on the amount of the used a priori information. 


\section{The testing of PDTT capabilities on a number of test objects.}

Modeling in finite element models with subsequent noising of surface temperature and discretization of data array with account of the parameters of the thermal imager (matrix size, temperature sensitivity) has been performed.

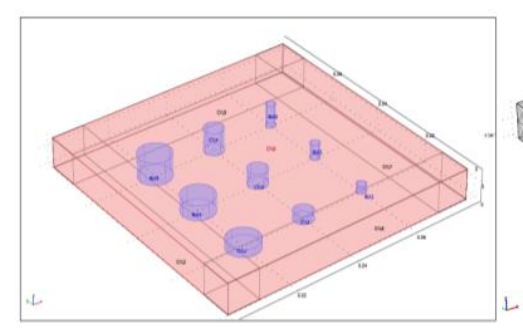

a

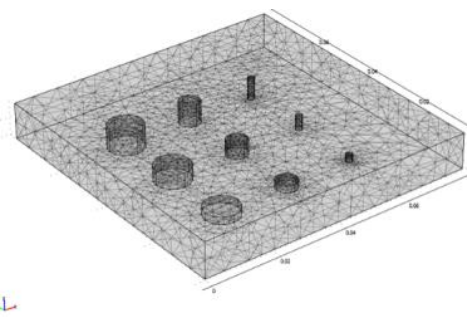

$b$

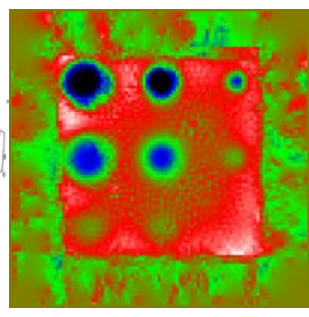

$c$

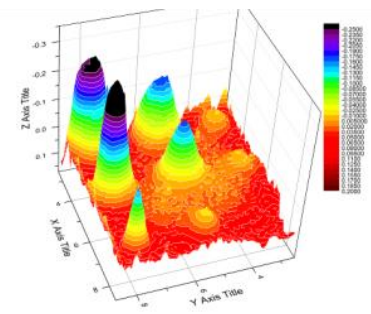

$d$

Fig. 3. Structure of the test object (a) and its division into finite elements (b). Results of preliminary decoding of the thermofilm using the PDTT method (for the first method of heating) and subsequent more accurate reconstruction (c, $d$ ). Only one of the available defects was not detected (diameter $4 \mathrm{~mm}$, thickness $2 \mathrm{~mm}$, depth $6 \mathrm{~mm}$ ).

In case of object heating from edges (heated support method), this minimal defect can be detected. Using the profiles drawn across the centers of defects the diameter of defects and the depth of their location and can be determined with accuracy at least $10 \%$ (for deep defects). Thus, it has been shown that the minimum ratio of the diameter of test defect to the depth of its location is at least 0,6 . At the same time, the accuracy of determining the defect diameter (using the inflection points) is at least $10 \%$ (figure 4).
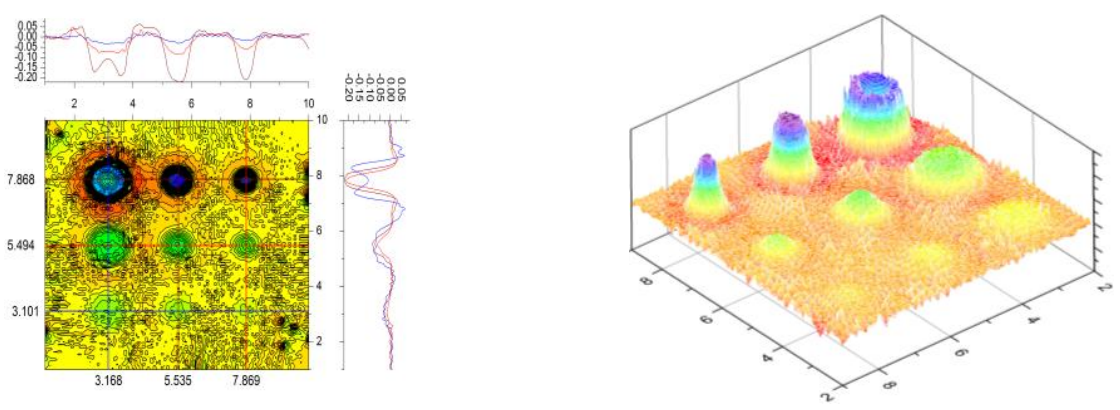

Fig. 4. Results of decoding of the thermofilm using the PDTT method (for the heated support method of heating)

The PDTT significantly reduces the region insensitive to thermal testing [4] and surpasses the capabilities of ultrasonic control (figure 5).

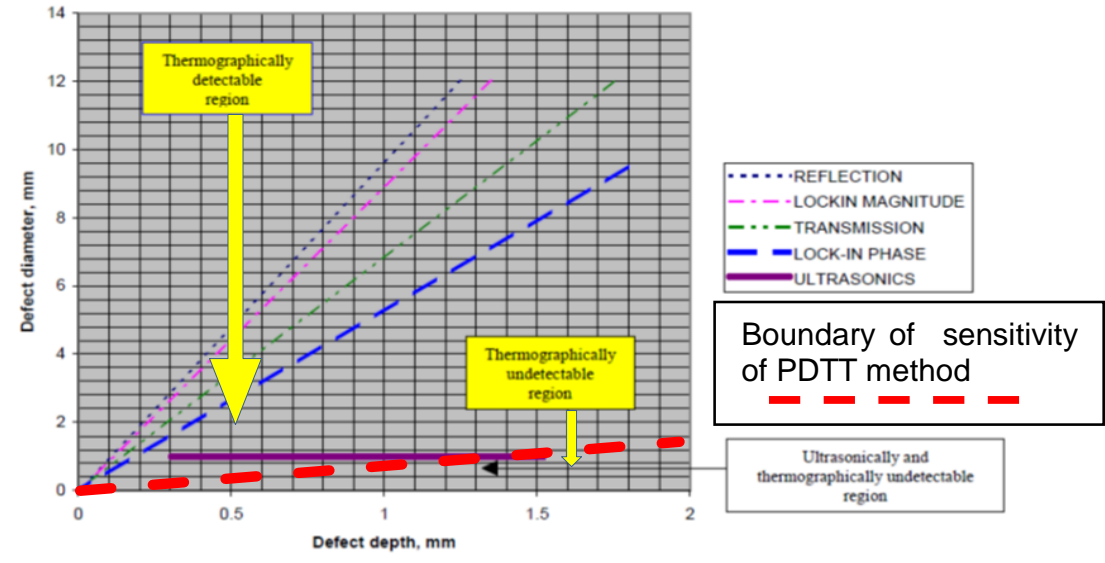

Fig.5. The PDTT method significantly expands the area of application of thermal imaging control and ensures a resolution comparable with ultrasonic control method. 


\section{The additional capabilities of the PDTT method}

The reconstruction of defect reveals not only the form of its internal boundaries, but also the internal hollowing. The reconstruction algorithm allows "looking" into the area shaded from the thermal flow (figure 6).
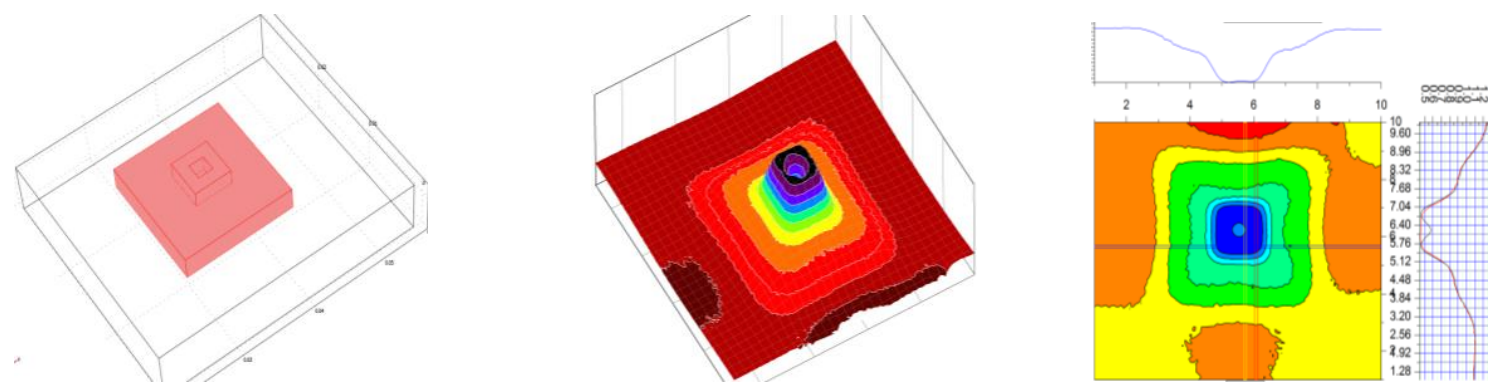

Fig.6. Absence of «shadow effects»

The PDTT method allows locally determining the value of the emissivity factor in each pixel of the thermogram with high accuracy and rerecording a thermofilm recorded by any thermal imager for realistic temperature distribution on the object's surface. According to the expert opinion, the natural inhomogeneity of the emissivity factor of various surfaces is up to $5 \%$. The presence of such "spots" - local areas of increased or decreased emissivity factor of the observed surface practically does not distort the results of reconstruction after their filtration using a special algorithm. Filtering of artifacts caused by fluctuations of the emitting capability of the surface of the object of control.

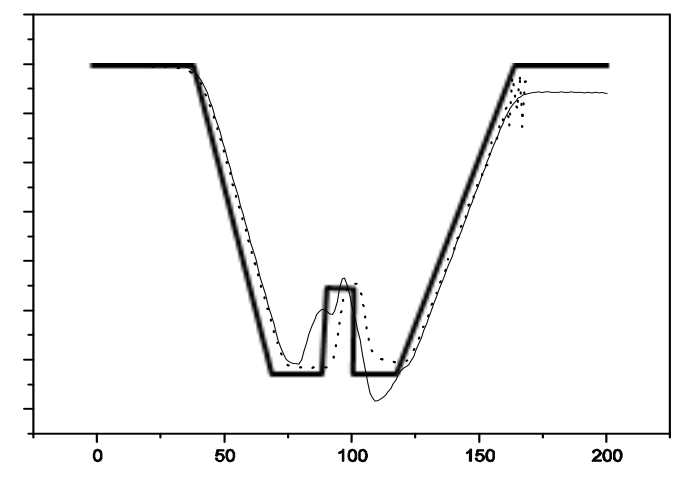

a

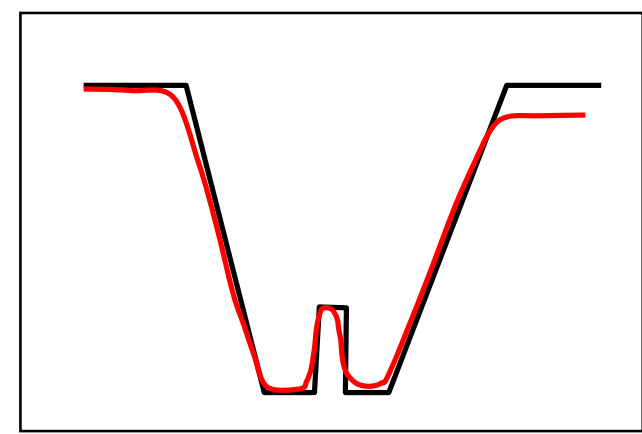

b

Fig.7. (a) reconstructed profile (thin solid line) in case of presence of the bell-shaped inhomogeneity of the emissivity coefficient with amplitude 1.05 between 80 and 120 pixels of the thermogram without using the algorithm of filtration,

(b) reconstructed profile using the algorithm of filtration (red line)

The PDTT method has low sensitivity to the parameter of temperature sensitivity, which allows using less expensive thermal imagers in most cases (figure 8).
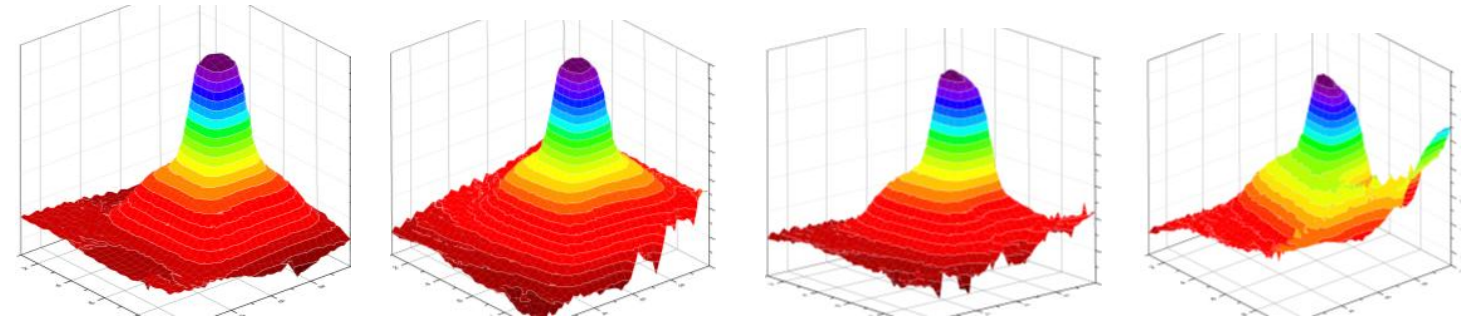

Fig.8. Results of reconstruction of irregular shape defect with the level of additive nose (with normal spectrum) $0.05 ; 0.1$; 0.2 u $0.4 K$. The temperature of dynamic heating of the observed surface in the defect area is up to $150 \mathrm{~K}$ in relation to the initial temperature. 
The temperature sensitivity of modern general-purpose thermal imagers is normally $0.06 \mathrm{~K}$. The temperature sensitivity of special-purpose thermal imagers for scientific research is $0.02 \mathrm{~K}$. The results of modeling show that the PDTT method is insensitive to temperature noises of this level (and even one order higher). Therefore, in a number of cases the requirements to temperature sensitivity can be decreased to $0.2 \mathrm{~K}$, which will allow using much less expensive thermal imagers in the control systems. Besides, it allows significantly decreasing the energy consumption for thermal control. The new PDTT method has a number of advantages of both scientific and commercial interest - this method is versatile and can be used with any method of creating thermal non-stationary flows.

\section{Possibilities of using PDTT for control of technical objects with irregular internal structure.}

The research of possibility of control of internal structure of cooling channels in high-pressure turbine blades using the PDTT method has been performed.

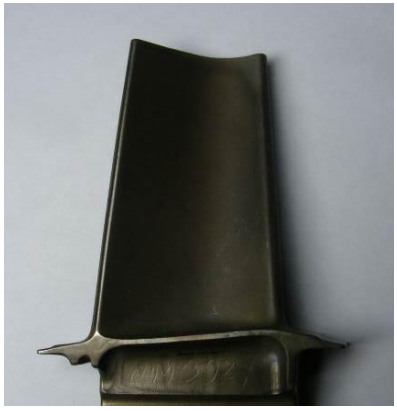

a

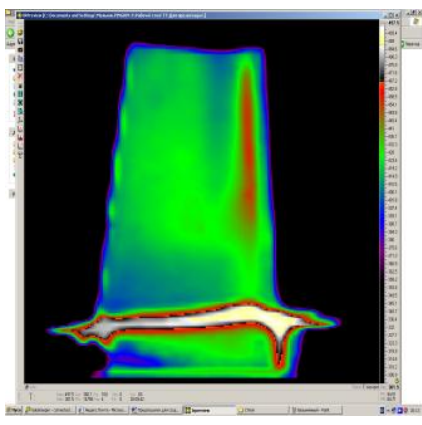

b

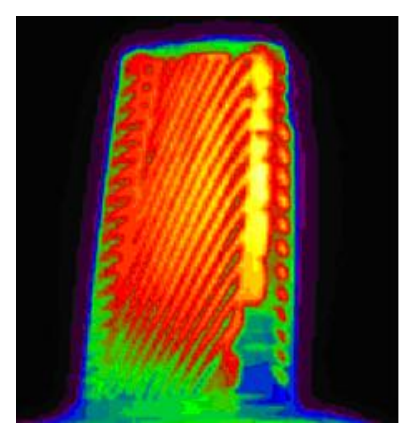

C

Fig.9. (a) Blade appearance; (b) sample shot from the thermofilm obtained in real thermal-imaging recording;(c) structure of blade internal channels (shot from the thermofilm of a blade with cut-off upper layer of metal)

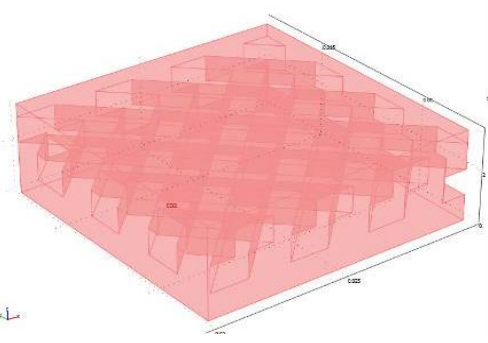

a

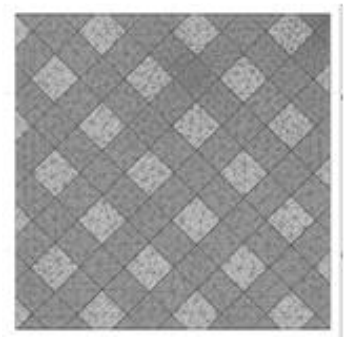

b

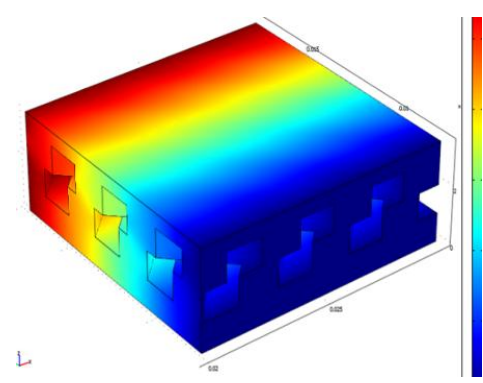

C

Fig.10. Model of blade element with two layers of cooling channels (a); structure of channels in the blade in the finite element model (plan view) (b) and results of numerical modeling of thermal flow in one of the horizontal directions (c)

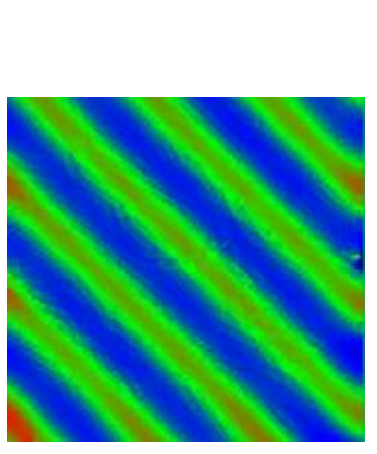

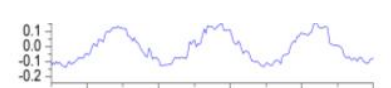

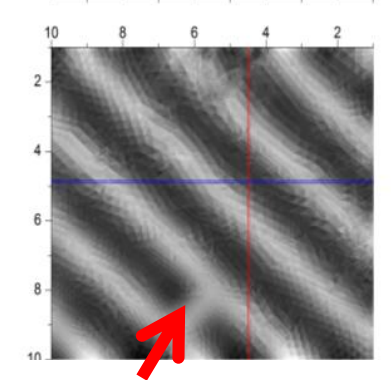

The accuracy of reconstruction of channel width (according to the inflection points) is at least 0.1 $\mathrm{mm}$. It is one order less than the depth of occurrence of the channels. reconstruction of the structure of channels using the refined algorithm (using the drawn cross-sections it is possible to determine the depth of location and width of channels with the accuracy of 0,1 $\mathrm{mm}$ at the depth of up to $2 \mathrm{~mm}$ );

Fig.11. (a) Results of reconstruction of the upper layer of channels (width $1 \mathrm{~mm}$, gap between the channels $1 \mathrm{~mm}$, depth of location $1 \mathrm{~mm}$ ); (b) profiles of reconstructed image in two cross-sections. Inclusion-type defect in one of the channels is reliably detected in the reconstructed image (red 
Variation of parameters of the reconstruction algorithm allows "focusing" the image on different depths in the object of control. The figure 12 illustrates the schematic layout of location of two layers of channels in the high-pressure turbine blade and a series of results of their reconstruction with smooth transition of "focusing" depth from the upper layer to the lower one. In case of increasing the depth of "focusing", the image of the upper layer of channels is distorted and superimposed on the image of the lower layer of channels, however the aggregate of images allows reconstructing a three-dimensional structure using the additional algorithm.
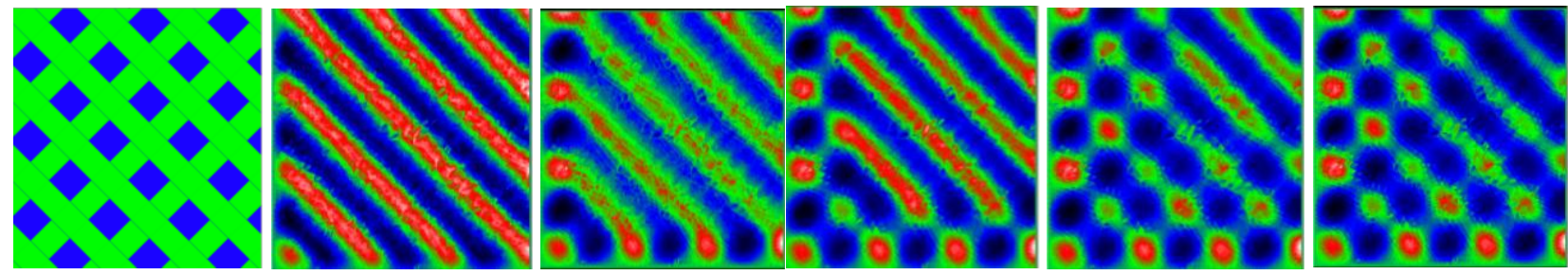

Fig.12. Reconstruction algorithm allows "focusing" the image on different depths in the object of control

\section{Conclusions}

The new PDTT method has a number of advantages of both scientific and commercial interest - this method is versatile and can be used with any method of creating thermal non-stationary flows. It is resistant to temperature noises in the initial thermogram up to $0.2 \mathrm{~K}$, which allows using less expensive IR cameras with sensitivity of at least $0.2 \mathrm{~K}$ in the control systems. It allows filtering artifacts relating to the inhomogeneity of the emitting capability (emissivity factor) of the observed surface with the value up to 5\%. The PDTT method allows detecting internal defects with size several times smaller than their depth of occurrence, which is one order better compared to alternative methods of DTT.

1. The PDTT method is applicable for control of objects with irregular internal structure.

2. The PDTT method allows reliably detecting the foreign inclusion-type defects and controlling with high accuracy the geometric parameters of the internal structure of objects.

3. The PDTT method is designed for using modern thermal imager with a standard set of parameters and does not require expensive additional equipment.

4. Preliminary numerical analysis of capabilities of the proposed method for specific objects and the parameters of expected defects is possible, indicating the requirements to the parameters of thermal imager and heating.

5. The PDTT method is superior in terms of information value to all alternative DTT methods and can also replace a number of more expensive control procedures using other methods (ultrasonic, x-ray, SHF).

Results of comparison of PDTT with other thermal control methods:

1. The PDTT method is additive - it allows adding new information to the already available data. The quality of reconstruction is determined by the set of thermal projections obtained for various directions of thermal flows in the object.

2. The PDTT method is resistant to temperature noises in the initial thermogram up to $0.2 \mathrm{~K}$, which allows using less expensive thermal imagers with sensitivity of at least $0.2 \mathrm{~K}$ in the control systems.

3. The PDTT method allows filtering artefacts relating to the inhomogeneity of the emitting capability (emissivity factor) of the observed surface with the value up to $5 \%$.

4. The PDTT method allows detecting internal defects with size several times smaller than their depth of occurrence, which is one order better compared to alternative methods of DTT.

5. The accuracy of measurement of geometric parameters of defects 10 times smaller than the depth of their occurrence can be achieved using special processing algorithms.

\section{REFERENCES}

[1] Vavilov V.P., "Thermal (infrared) tomography: terminology, basic procedures and applications to nondestructive testing of composite materials", Defectoscopiya (Rus. J. NDT), No.3, p. 3-15, 2010.

[2] G. Busse, "Nondestructive evaluation of polymer materials," NDT \&E Int'l, 27, 253 (1994).

[3] V. Vavilov, D. Nesteruk, V. Shirayev, A. Ivanov, Some novel approaches to thermal tomography of CFRP composites, QIRT 2010-145

[4] B. S. Wong, C. G. Tui, W. Bai, P. H. Tan, B. S. Low, and K. S. Tan, "Thermographic Evaluation of Defects in Composite Materials," Insight: Non-Destructive Testing and Condition Monitoring, Vol. 41, No. 8, pp. 504, 1999.

[5] Steven M Shepard, Flash Thermography of Aerospace Composites 2 . Thermographic Signal Reconstructioning Quality (2007)

[6] Melnyk S. Thermal tomography on the basis of an information method // QIRT 2004-028 C.6.1-C.6.6 